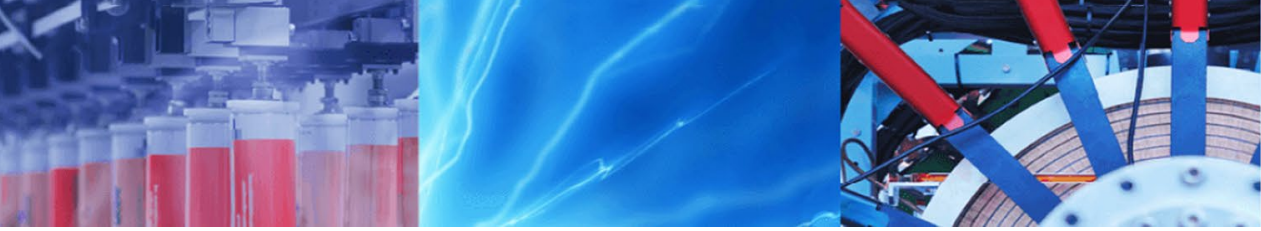

Research Article

\title{
Photoelectronic properties of composite films based on conductive polymer PIDT-BT and single-walled carbon nanotubes
}

\author{
Andrey N. Aleshin ${ }^{1} \cdot$ Baode Zhang ${ }^{2,3} \cdot$ Petr A. Aleshin $^{1} \cdot$ Vasily N. Petrov ${ }^{1} \cdot$ Irina N. Trapeznikova ${ }^{1} \cdot$ Günter Reiter $^{3}$. \\ Janis Kleperis ${ }^{4}$
}

(c) Springer Nature Switzerland AG 2019

\begin{abstract}
We investigated photoelectronic properties of composite films based on semiconducting polymer poly(indacenodithiophene-co-benzothiadiazole) (PIDT-BT) and single-walled carbon nanotubes (SWCNTs). It was found that the absorbance spectra of PIDT-BT and PIDT-BT/SWCNT (1:0.3) films were in the spectral region from 200 to $800 \mathrm{~nm}$, with an absorbance edge at ca. $1.71 \mathrm{eV}$. Adding of ca $30 \mathrm{wt} \%$ of SWCNT to the PIDT-BT polymer matrix resulted in a decrease in absorbance by a factor of 1.5. We made a hole-only device based on PIDT-BT lightly doped with carbon nanotubes. Current-voltage characteristics (I-Vs) of PIDT-BT/SWCNT (1:0.3) composite films were measured within temperature interval from 300 to $77 \mathrm{~K}$ in the dark and under illumination by a solar simulator. I-Vs demonstrated an increase in photocurrent under illumination at $300 \mathrm{~K}$ by factor of ca. 1.4-1.7 with respect to dark $I-V$ s. The dark $I-V s$ were investigated in the temperature interval from 300 to $77 \mathrm{~K}$. It was found that the temperature dependence of the resistivity of PIDT-BT/SWCNT (1:0.3) films followed a power law in this temperature region. It has been established that the main charge carrier transport mechanism is the space charge limited current mechanism in the dark and the space charge limited photocurrents under illumination.
\end{abstract}

Keywords Semiconducting polymers · Single-walled carbon nanotubes · Charge transport - Temperature dependence of resistivity $\cdot$ Photoelectronic properties

\section{Introduction}

Recent developments of semiconducting polymer-inorganic hybrid nanocomposites have attracted the interest of scientists from both fundamental and applied points of view, since such nanocomposites are used in various photoelectronic applications [1]. This is due to the unique properties of such hybrid nanocomposites, namely flexibility, adaptability, the ability to print over a large area and their lightweight, along with noticeable electrical conductivity, low thermal conductivity, which can facilitate their use in solar cells, photodetectors and field-effect transistors. It has been shown that the introduction of single-walled carbon nanotubes (SWCNTs) into the conducting polymer matrix improves the electrical conductivity, as well as the mechanical properties of the neat polymers [2]. SWCNTs have a unique structure, narrow distribution in size, high available surface area, low resistivity and high stability [3]. Recent research on CNTs indicates it is a potential material for the organic and hybrid solar cells $[4,5]$. Namely, CNTs have a remarkably high level of electronic mobility (much higher than the hole mobility of semiconducting polymers) and work function similar to that of the electrodes (Au or ITO) which makes them an

$\triangle$ Andrey N. Aleshin, aleshin@transport.ioffe.ru; $₫$ Baode Zhang, baode.zhang@physik.uni-freiburg.de; zhangbaode@lnpu.edu.cn | ${ }^{1}$ Ioffe Institute, Saint-Petersburg, Russia 194021. ${ }^{2}$ School of Chemistry and Material Science, Liaoning Shihua University, Fushun 113001, People's Republic of China. ${ }^{3}$ Institute of Physics, Albert-Ludwig-University of Freiburg, Hermann-Herder-Str. 3, 79104 Freiburg, Germany. ${ }^{4}$ Institute of Solid State Physics, University of Latvia, Riga 1063, Latvia. 
ideal candidate for the usage in the hole-extraction layer (or hole transporting layer) in organic photovoltaics [6, 7]. Investigation of SWCNT-polymer solar cells has been conducted toward developing alternative, lightweight, flexible devices for space power applications [5]. Benzothiadiazole (BT)-based polymers such as PIDT-BT with indacenodithiophene (IDT) as the donor $(D)$ and BT as the acceptor $(A)$ unit have been recognized as one of the first successful BT-based $D-A$ copolymers with a mobility exceeding $1 \mathrm{~cm}^{2} / \mathrm{Vs}$ [8-10]. Thin films of PIDT-BT exhibited low crystallinity with a large $\pi$-stacking distance and faceon orientation. A high hole mobility up to $1.25 \mathrm{~cm}^{2} / \mathrm{Vs}$ was achieved in bottom-contact/top-gate field-effect transistors [8]. Photoelectronic properties of both, PIDT-BT and SWCNTs, were investigated well during the last years $[2,3$, $8-10]$. Both carbon nanotubes and semiconducting polymers, such as PIDT-BT, possess conjugated $\pi$-systems, and the nature of their electronic interaction is anticipated to occur via $\pi-\pi$ stacking. At the same time, the use of holeonly devices such as PIDT-BT lightly doped with CNT with gold electrodes is of important for mobility measurements. However, the doping effect of PIDT-BT semiconducting polymer films by the introduction of SWCNTs has not yet been investigated. In addition, the electronic properties and the transport mechanism of composite films based on the PIDT-BT semiconducting polymer weakly and medium doped of SWCNT have not been studied in detail at temperatures up to $77 \mathrm{~K}$.

In this work, we obtained films with a hole-only conduction based on the PIDT-BT semiconductor polymer, with weak and intermediate doping with single-walled carbon nanotubes, and investigated their optical and electrical properties in order to clarify the mechanism of charge carrier transport in such composite films. The obtained films were characterized by absorbance, atomic force microscope and current-voltage characteristics (I-Vs) techniques. Adding of ca. $30 \mathrm{wt} \%$ of SWCNTs to the PIDTBT matrix resulted in a decrease in absorbance by factor of 1.5. The results of measurements of the $I-V \mathrm{~s}$ of PIDTBT/SWCNT composite films (1:0.3) in the dark and under illumination at temperatures of $300-77 \mathrm{~K}$ show that the charge carrier transport is determined by the mechanism of the space charge limited currents in the dark and by the space charge limited photocurrents under illumination, and the temperature dependence of the resistivity of such films obeyed a power law.

\section{Experimental}

To prepare samples, the conjugated polymer PIDT-BT with a band gap $E_{\mathrm{g}} \approx 1.71 \mathrm{eV}$ [10] and SWCNTs (obtained from Sigma-Aldrich with the following parameters: $98 \%$ semiconducting, $2 \%$ metallic, with the length ca. $0.3-5 \mu \mathrm{m}$, diameter of ca. $1.2-1.7 \mathrm{~nm}$, a density of ca. $1.7-1.9 \mathrm{~g} / \mathrm{cm}^{3}$ ) and used without additional treatment. Both components were dissolved and dispersed separately in chloroform. Subsequently, the solutions were mixed with relative amounts of 1:0.1 and 1:0.3 and subjected to ultrasonic treatment for 10 min on a Bandeline Sonopuls HD 2070 ultrasonic mixer (at a frequency $f \approx 20 \mathrm{kHz}$ ). The resulting solution was applied by drop-cast technique at a temperature of $300 \mathrm{~K}$ in an atmosphere of $\mathrm{N}_{2}$ on glass substrates with thermally evaporated gold $(\mathrm{Au})$ electrodes on top. (We used the usual lithography technique to obtain an electrode spacing of $\approx 15 \mu \mathrm{m}$.) Afterward, the films were dried at $100^{\circ} \mathrm{C}$ under $\mathrm{N}_{2}$ for $10 \mathrm{~min}$ and kept in vacuum desiccator (MB SANPLATEC Corp.) until measurements. Thus, in our study, we are dealing with films with hole-only conductivity based on PIDT-BT, with weakly and intermediate doped with SWCNT, which are referred to as composite films in the text. The morphology of the PIDT-BT/ SWCNT composite films was investigated by atomic force microscope (AFM) - Solver P47-NT-MDT. The thickness of the films obtained by drop casting onto quartz substrates was $\approx 1 \mu \mathrm{m}$, according to the AFM data. The chemical structure of PIDT-BT/ SWCNT and the schematic morphology of the PIDT-BT/SWCNT nanocomposite films are shown in Fig. 1a-c, respectively. Absorbance spectra of the films deposited by drop-casting technique onto quartz substrates were studied using a Cary-50 (Varian) spectrometer. The DC I-Vs of the PIDT-BT and PIDT-BT/SWCNT films were measured in nitrogen atmosphere in the dark and under solar simulator illumination at temperatures from 300 to $77 \mathrm{~K}$ using a sample holder of a liquid $\mathrm{N}_{2}$ cooled optical cryostat optCRYO198 with temperature stabilization and DC electronic computer-controlled measuring system based on a Keithley 6487 picoamperemeter/ voltage source. The DC voltages were varied from -10 to $+10 \mathrm{~V}$. The electrical contacts for the samples were made with a silver wire, which was fixed to the metal electrodes with a silver paste.

\section{Results and discussion}

The results of AFM investigations of the PIDT-BT/SWCNT (1:0.3) film are shown in Fig. 2a-c. As can be seen from these figures, the surface of PIDT-BT/SWCNT nanocomposite films was moderately rough. We found that the overall height variation was about $50-100 \mathrm{~nm}$, exhibiting steps of several nm (Fig. 2b), which may reflect SWCNTs and their agglomerates covered with the PIDT-BT polymer. It was found that the average roughness and root-mean-square (RMS) values of the PIDT-BT/SWCNT film (1:0.3) as shown in Fig. $2 \mathrm{a}-\mathrm{c}$ are about $8.8 \mathrm{~nm}$ and $11 \mathrm{~nm}$, respectively. These 
(a)

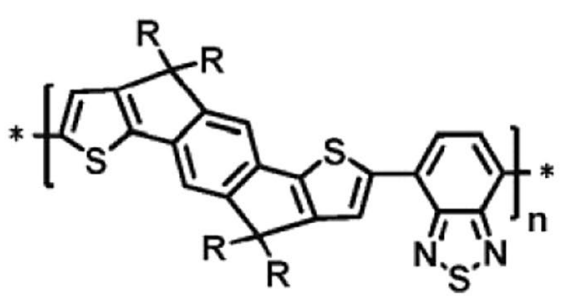

$\mathrm{R}=$ 4-n-hexylphenyl

(b)

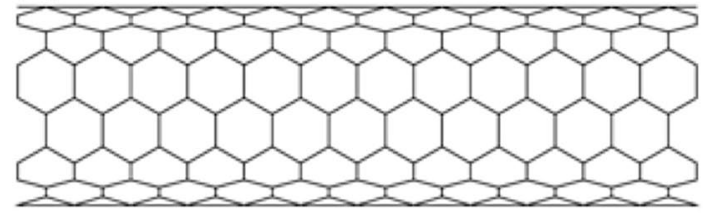

(c)

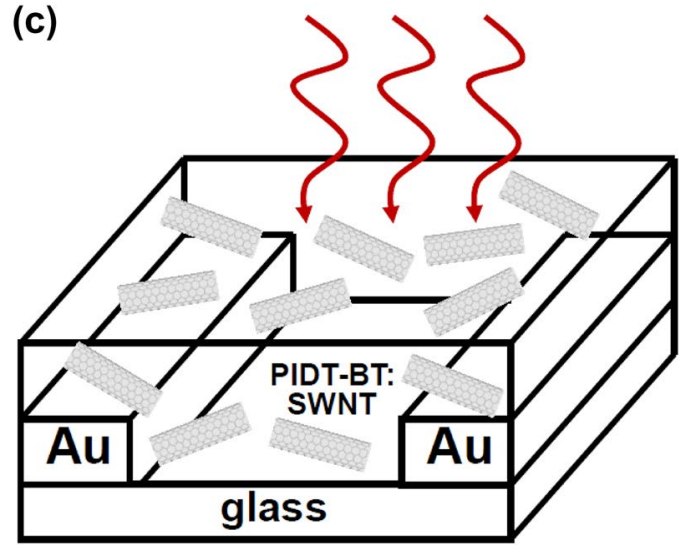

Fig. 1 Chemical structure of PIDT-BT polymer, $R=4$-n-hexylphenyl, $\mathrm{C}_{16} \mathrm{H}_{33}(\mathbf{a})$, SWCNT (b) and schematic drawing of the morphology of the PIDT-BT/SWCNT nanocomposite film (c)

parameters are higher than that of a pure PIDT-BT film, where the average roughness and root mean square are $4 \mathrm{~nm}$ and $5 \mathrm{~nm}$, respectively (not shown). As can be seen from our AFM measurements, the modification of the PIDTBT polymer with SWCNT led to a relatively smooth surface of the PIDT-BT/SWCNT composite films.

Figure 3 shows typical absorbance spectra of neat PIDTBT (1) and PIDT-BT/SWCNT (1:0.3) films (2) deposited onto quartz substrates. As can be seen from Fig. 3, the absorbance spectra of PIDT-BT and PIDT-BT/SWCNT (1:0.3) films were in the spectral region from $200 \mathrm{~nm}$ to $800 \mathrm{~nm}$. It was found that the absorbance onset for PIDT-BT and PIDT-BT/ SWCNT (1:0.3) films is about $725 \mathrm{~nm}$, which corresponds to $1.71 \mathrm{eV}$, which correlates well with the band gap of the PIDT-BT polymer: $\mathrm{Eg} \approx 1.71$. eV [8]. As evident from Fig. 3, adding of about 30 wt $\%$ of SWCNTs to the PIDT-BT polymer matrix resulted in a decrease in absorbance by a factor of 1.5 without changing of absorbance maxima (at $\sim 688 \mathrm{~nm}$ and $\sim 417 \mathrm{~nm}$ ) and absorbance edge position.
The typical I-Vs of PIDT-BT/SWCNT (1:0.3) composite film measured in the dark and under illumination by solar simulator at $300 \mathrm{~K}$ are shown in Fig. $4 \mathrm{a}, \mathrm{b}$. It is found that photocurrent of PIDT-BT/SWCNT (1:0.3) film at $300 \mathrm{~K}$ increased by factor $\approx 1.5-1.7$ under solar simulator illumination with respect to dark $I-V$ s. There was some small hysteresis of $I-V s$ of composite film at $300 \mathrm{~K}$ in the dark probably due to the influence of shallow traps in the polymer matrix. However, as can be seen from Fig. $4 a$, b, this small hysteresis of $I-V$ s disappeared for negative voltages under and after solar simulator illumination since all traps were populated (Fig. 4a, curves 2 and 3). This reproducible hysteresis of $I-V s$ in PIDT-BT/SWCNT films is very similar to that observed at $300 \mathrm{~K}$ in composite films based on SWCNTs embedded in poly(methyl methacrylate) (PMMA) [11]. As in the case of PIDT-BT/SWCNT films, a stable hysteresis loop along anticlockwise direction at positive and negative biases was observed in PMMA/SWCNT films and the width of the hysteresis was reduced or quenched when the film was exposed to light [11]. By analogy with PMMA/ SWCNT composite films, the observed $I-V$ s hysteresis effects of the PIDT-BT/SWCNT films have been attributed to the injection of electrons from the electrodes to the trap sites located in the PIDT-BT/SWCNT layer.

Inset 1 in Fig. 4b shows lg-lg plots for positive voltages for the same sample before and under illumination. As can be seen from Inset 1 , the dark I-Vs at low voltages are linear, but not ohmic, which is characteristic of the mechanism of space charge limited currents (SCLC) $[12,13]$. The same behavior was found for the I-Vs of this sample when illuminated by a solar simulator, which means that the mechanism of space charge photocurrents is the main transport mechanism in lighting [14]. For comparison, we measured the $I-V$ s of a neat PIDT-BT polymer film at $300 \mathrm{~K}$ deposited onto a glass substrate under the same conditions as the PIDT-BT/SWCNT (1:0.3) film. Inset 2 in Fig. 4b shows $I-V$ s of neat PIDT-BT film in the dark and under solar simulator illumination. As evident from Inset 2 in Fig. $4 \mathrm{~b}$, the DC conductivity of the neat PIDT-BT polymer film was lower by two orders of magnitude compared with PIDTBT/SWCNT (1:0.3) film. The dark current of pure PIDT-BT is very low, but it increases by two orders of magnitude after illumination with a solar simulator. Higher, but also very low DC conductivity was also found for PIDT-BT/SWCNT (1:0.1) composite films (not shown).

Figure 5 presents $I-V s$ of the PIDT-BT/SWCNT (1:0.3) composite film measured in the dark at different temperatures within temperature interval from 300 to $77 \mathrm{~K}$. As evident from Fig. 5, the magnitude of DC current went down gradually as the sample temperature decreased from $300 \mathrm{~K}$ down to $77 \mathrm{~K}$. The resistivity at each temperature was estimated from the linear regime of the $I-V s$ at voltages varied from +1 to $-1 \mathrm{~V}$. It should be noted that, from Inset 1 in 

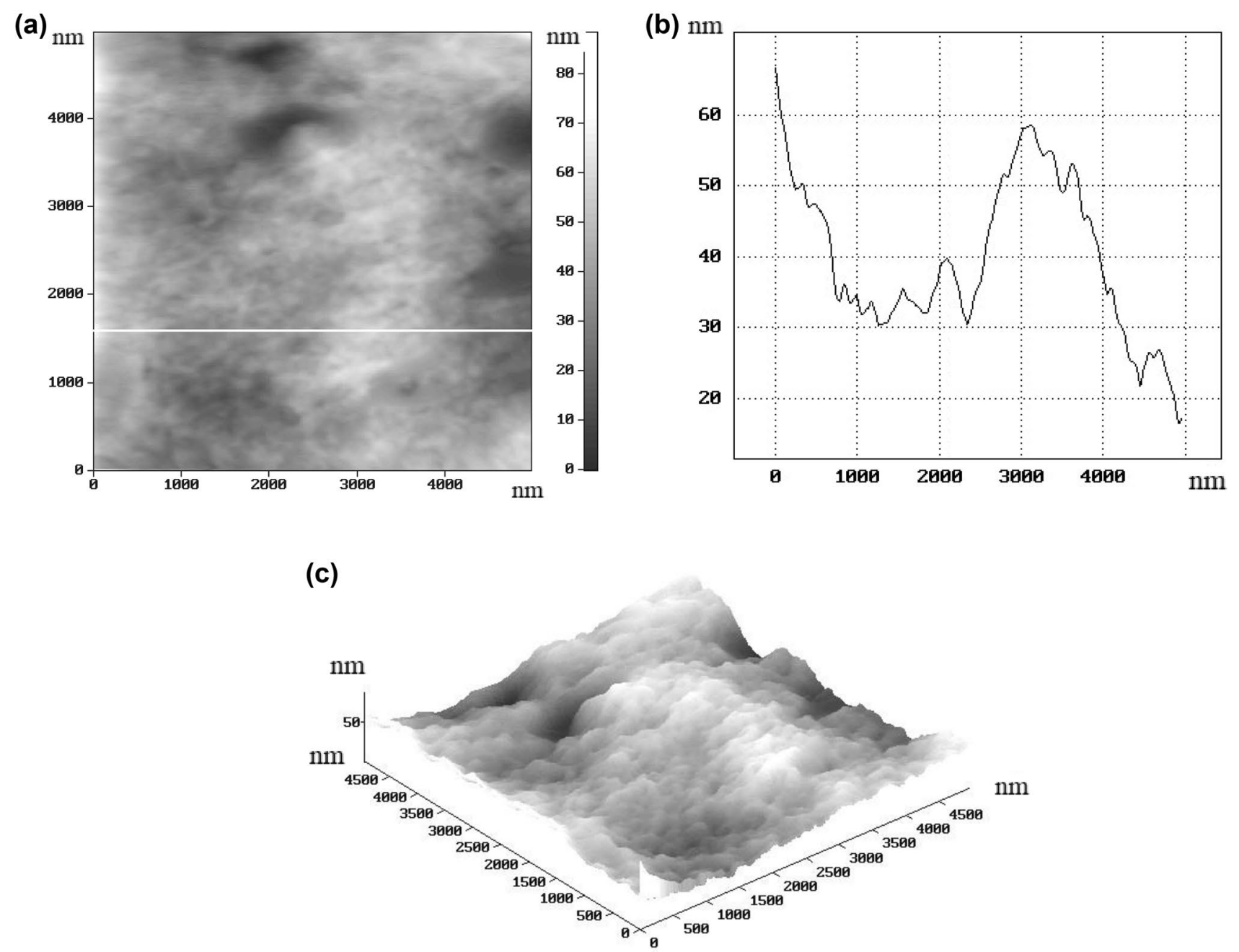

Fig. 2 Results of AFM investigations of the surface of PIDT-BT/SWCNT (1:0.3) films

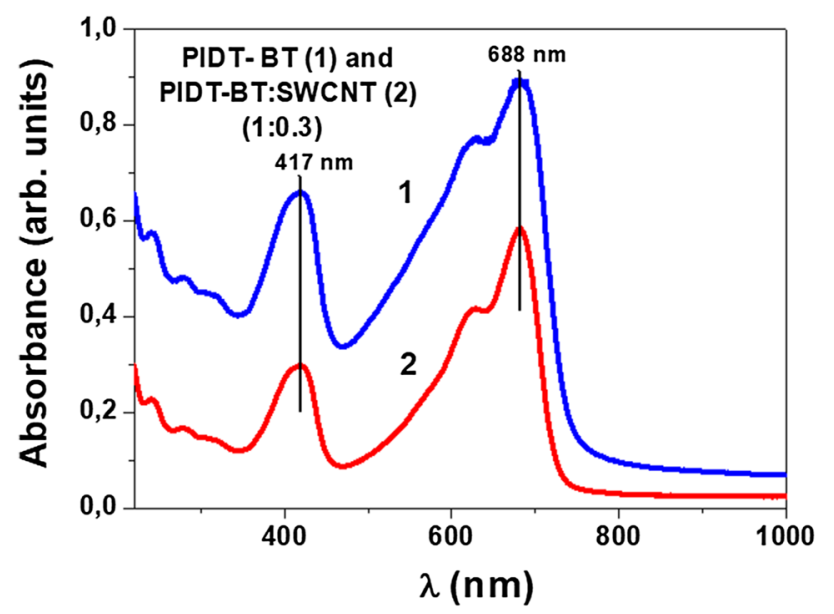

Fig. 3 Absorbance spectra of pure PIDT-BT (1) and PIDT-BT/SWCNT (1:0.3) (2) films
Fig. $4 b$, in fact, the linear $I-V$ mode in a device with one type of carrier is not ohmic and obeys the dependence $1-V^{x}$, where $x>2$, which is characteristic of the SCLC mechanism $[15,16]$. The temperature dependence of the resistivity $\rho(\mathrm{T})$ of the PIDT-BT/SWCNT (1:0.3) nanocomposite film is shown in Fig. 6 . Inset in Fig. 6 demonstrates the $\rho(T)$ vs. $10^{3} / T$ dependence for the same sample (curve 1) and for the sample PIDT-BT/SWCNT (1:0.1) (curve 2). We could not measure the $\rho(T)$ dependence of the pure PIDT-BT film due to the very high resistance of the sample and insufficient sensitivity of our equipment. As can be seen from Fig. 6 and the inset, the $\rho(T)$ dependence of PIDT-BT/SWCNT (1:0.3) film has the nature of an activated process but did not exhibit a constant activation energy within the entire temperature interval. Unlike the PIDT-BT/SWCNT sample (1:0.3), the $\rho(T)$ dependence for the PIDT-BT/SWCNT sample (1:0.1) (curve 2 in Fig. 6) is very strong and shows only constant activation energy. In fact, for temperatures from 300 to $280 \mathrm{~K}$ for the sample 2 and from 300 to $250 \mathrm{~K}$ for the 


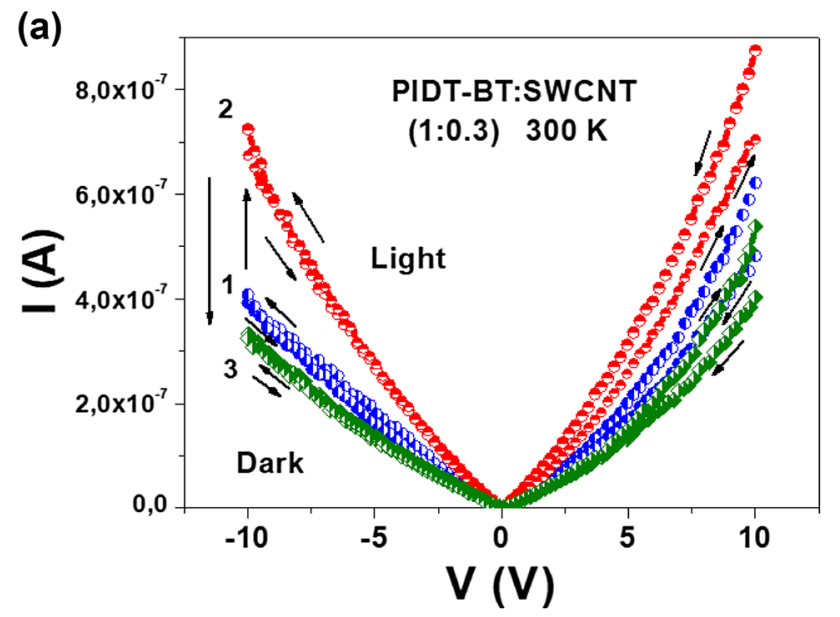

(b)

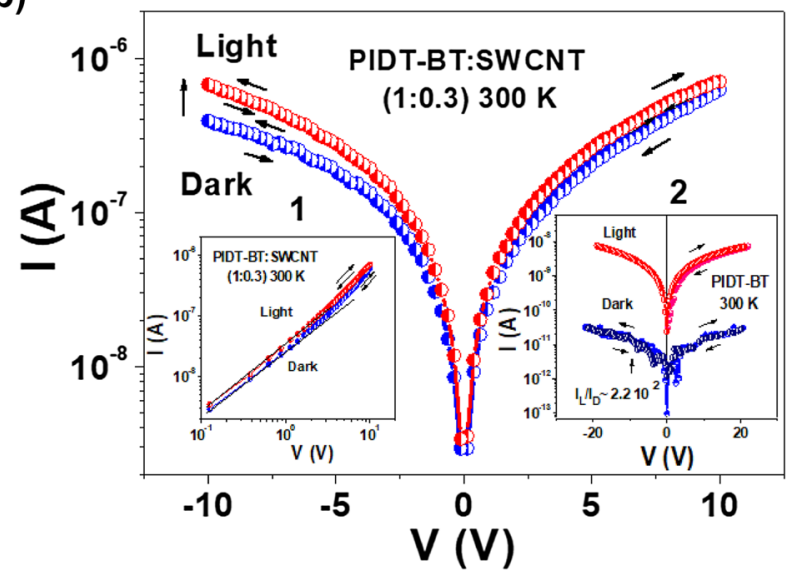

Fig. 4 a $I-V$ s of PIDT-BT/SWCNT (1:0.3) nanocomposite film measured in the dark before illumination (curve 1), under illumination (curve 2) and after illumination (curve 3 ) at $300 \mathrm{~K} ; \mathbf{b} \mathrm{lg} / \mathrm{vs}$. $V$ for the same sample before and under illumination. Inset 1 in (b) shows the log-log graphs for positive voltages for the same sample at $300 \mathrm{~K}$. Inset 2 in (b): $1-V$ s of neat PIDT-BT film at $300 \mathrm{~K}$ under the same conditions

sample 1, the behavior can be described by an exponential dependence:

$\rho(T)=\rho_{0} \exp \left(E_{\mathrm{act}} / k_{\mathrm{B}} T\right)$

where $E_{\text {act }}$ is the activation energy, $T$ is the temperature, and $k_{\mathrm{B}}$ is the Boltzmann constant. $E_{\mathrm{act}}$ can be calculated from the following equation:

$E_{\text {act }}(\mathrm{meV})=(200 \Delta \lg \rho) /(\Delta 1000 / T)$

According to our estimates, $E_{\text {act }}$ for the PIDT-BT/SWCNT (1:0.1) film at $T=300-280 \mathrm{~K}$ and for the PIDT-BT/SWCNT (1:0.3) film at $T=300-250 \mathrm{~K}$ were found to be $E_{\text {act }} \approx 1.95 \mathrm{eV}$ and $E_{\text {act }} \approx 62 \mathrm{meV}$, respectively. However, at lower temperatures, a transition of $\rho(T)$ to a power law regime $\rho(T) \sim T^{-m}$ for the PIDT-BT/SWCNT (1:0.3) film was observed. One can expect a transition in $\rho(T)$ to a variable-range hopping

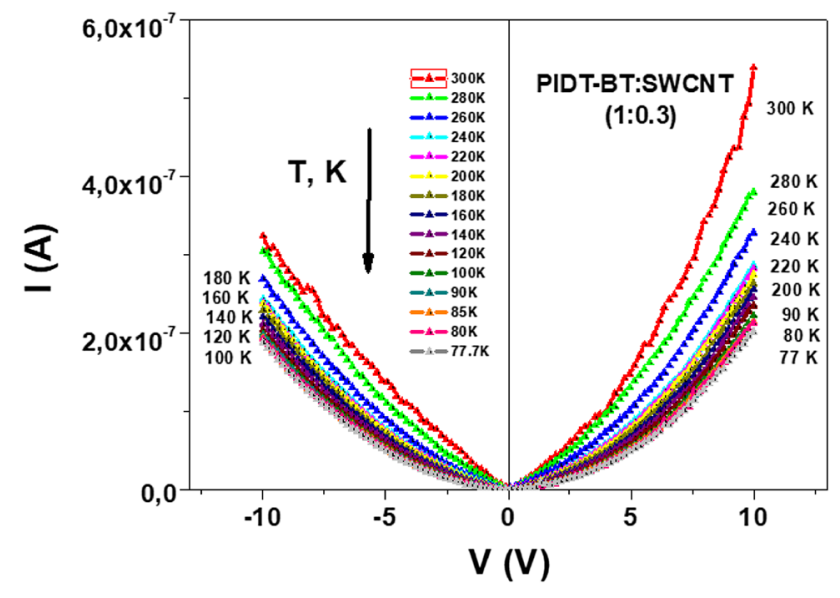

Fig. 5 I-Vs of the PIDT-BT/SWCNT (1:0.3) nanocomposite film measured in the dark at different temperatures within the temperature interval from 300 to $77 \mathrm{~K}$ at $T[\mathrm{~K}]=300,280,260,240,220,200,180$, $160,140,120,100,90,85,80,77$

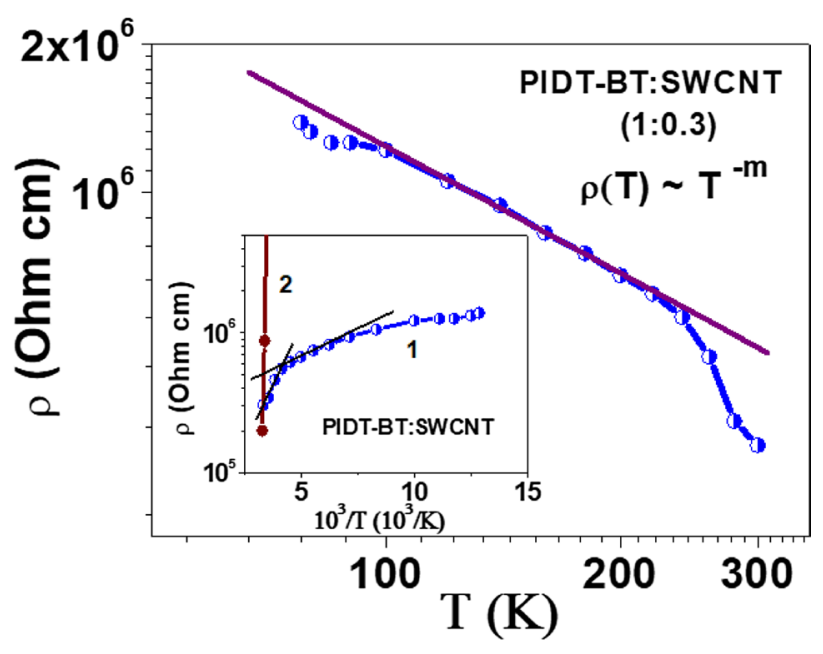

Fig. 6 Temperature dependence of the resistivity of the PIDT-BT/ SWCNT (1:0.3) nanocomposite film. Inset: $\rho(T)$ vs. $10^{3} / T$ dependences for the same PIDT-BT/SWCNT (1:0.3) sample (curve 1) and for PIDT-BT/SWCNT (1:0.1) (curve 2) nanocomposite film

(VRH) transport regime described by the dependence: $\rho(T)=\rho_{0} \exp \left(T_{0} / k_{B} T\right)^{p}$.

In our opinion, in the case of the PIDT-BT/SWCNT (1:0.1) nanocomposite film, the main transport mechanism is the thermal activation of carriers from HOMO to LUMO, which can also be expected in the film of pure PIDT-BT due to the observed $E_{\text {act }} \approx 1.95 \mathrm{eV}$ which is of the same order as the energy gap of the PIDT-BT polymer (but not exactly the same). Thus, we can assume that the $\rho(T)$ dependence of the pure PIDT-BT film should be somewhat similar to the dependence obtained for the PIDT-BT/SWCNT film (1:0.1). However, in case of PIDT-BT/SWCNT (1:0.3) 
nanocomposite films, the transport mechanism is more complex, consisting of several mechanisms dominating at different temperature intervals. In particular, transport should involve a thermally activated hopping mechanism at high temperatures. At intermediate temperatures, the effect of barriers to charge injection can be significant. It is worth noting that we used gold electrodes to inject holes into our device with one type of charge carriers. Since the $\mathrm{HOMO}$ energy level is deeper than the work function of $\mathrm{Au}$, it can be expected that the energy barriers to charge injection are about $100 \mathrm{meV}$ or more. The observed activation energy of the $\rho(T)$ dependence correlates with this energy [17]. A VRH transport regime might be possible at temperatures below $77 \mathrm{~K}$ [18-20]. The low-temperature charge transport in single SWCNT is the subject of intensive discussion [21]. However, such low-temperature experiments require particularly careful sample preparation. Usually, the VRH model is valid mostly at low temperatures. Thus, one may suggest that the tunneling model and its derivatives are more appropriate in the case of low-temperature transport of PIDT-BT/SWCNT composite films below $77 \mathrm{~K}$.

Figure $7 \mathrm{a}, \mathrm{b}$ demonstrates the $I-V$ s of PIDT-BT/SWCNT (1:0.3) composite film measured in the dark and under illumination by a solar simulator at $77 \mathrm{~K}$. As evident from Fig. 7a,b, the photocurrent of the PIDT-BT/SWCNT (1:0.3) film at $77 \mathrm{~K}$ increased by factor of ca. 1.4 under illumination by a solar simulator with respect to dark $I-V \mathrm{~s}$, similar to the behavior observed for the same sample at room temperature. As shown in Inset 1 in Fig. $7 \mathrm{~b}, I-V$ s at $77 \mathrm{~K}$ at low voltages are not ohmic; they follow the dependences $I-V^{x}$, where $x>2$ in the dark and under illumination, which is typical for the SCLC mechanism $[15,16]$ similar to $I-V s$ for the same sample measured at $300 \mathrm{~K}$. In contrast to our results at $300 \mathrm{~K}$, there is no hysteresis $I-V s$ in the dark and under illumination at $77 \mathrm{~K}$, so when the PIDT-BT/ SWCNT composite film (1:0.3) cools down to $T \approx 77 \mathrm{~K}$, the transport mechanism responsible for the hysteresis $I-V \mathrm{~s}$ is suppressed (this may be traps and the ionic conductivity typical of semiconductor polymers at room temperature [18]) in the PIDT-BT polymer matrix (probably due to $R=\mathrm{C}_{16} \mathrm{H}_{33}$ ), achieving a rather balanced charge transport in hybrid PIDT-BT/SWCNT structure. One can conclude that photoelectronic properties of composite films based on conductive polymer PIDT-BT modified with SWCNT remained similar (in the dark and under solar simulator illumination) within the temperature range from 300 to $77 \mathrm{~K}$ and the transport mechanism is determined by SCLC when measured in the dark and by space charge limited photocurrents when measured under illumination.

The details of charge carrier transport in the Au/PIDT-BT/ SWCNT/Au structures can be understood from the energy diagram as shown in Fig. 8. (This energy diagram is based on results published in references $[6,20]$.) As evident from

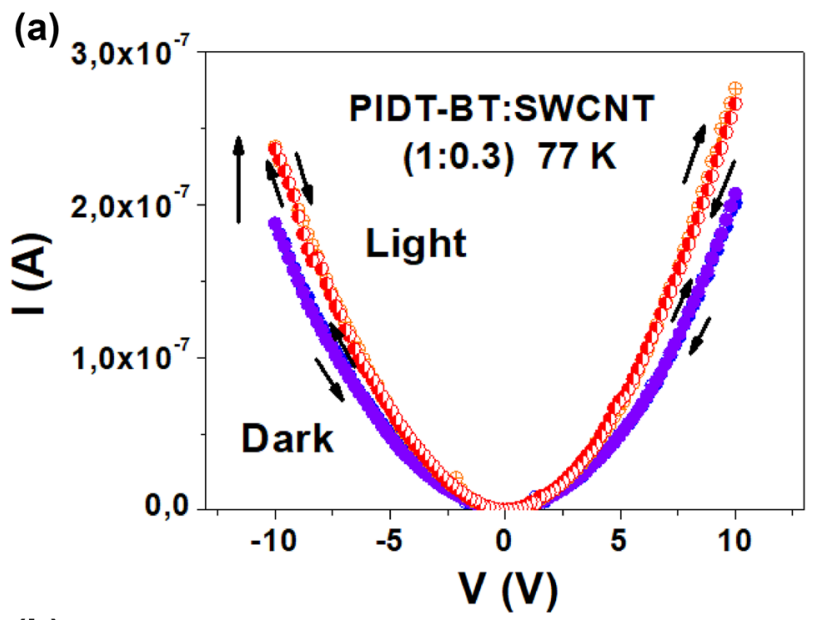

(b)

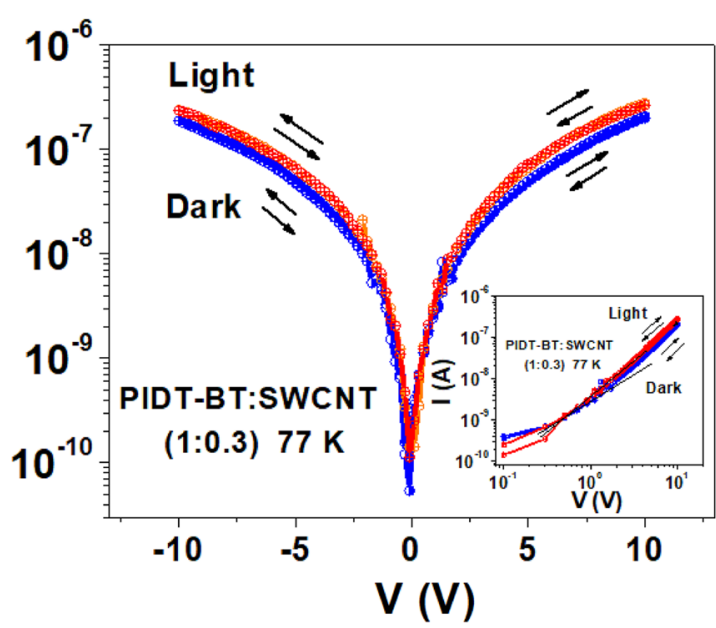

Fig. 7 I-Vs of PIDT-BT/SWCNT (1:0.3) nanocomposite film measured in the dark and under illumination at $77 \mathrm{~K}$. Inset shows the $\mathrm{lg}-\mathrm{lg}$ plots for positive voltages for the same sample at $77 \mathrm{~K}$

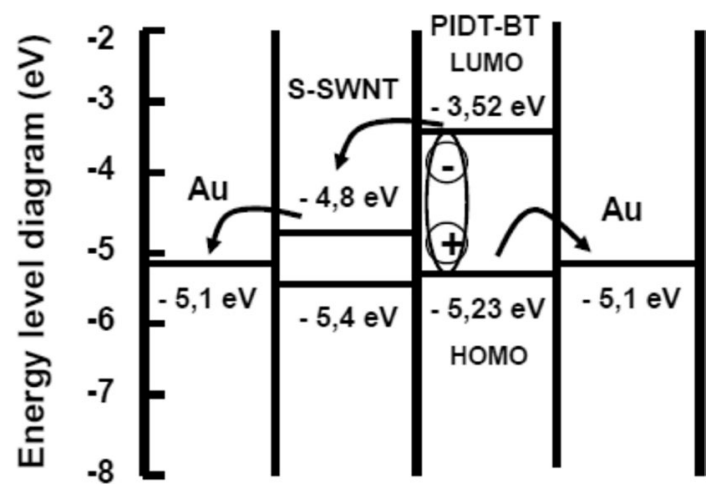

Fig. 8 Band diagram of the Au/PIDT-BT/SWCNT/Au structure

Fig. 8, excitons are generated in the PIDT-BT polymer, and holes are collected at the Au contact. Electron transfer is possible through the conduction band of the semiconducting SWCNTs. In our case, one may suggest that the 
semiconducting SWCNTs played a role as an electronconducting network. However, as evident from our estimation of the activation energy, PIDT-BT/SWCNT (1:0.3) film at temperatures $300-250 \mathrm{~K}$, the obtained value of $E_{\text {act }} \approx 62 \mathrm{meV}$ is much lower than the one expected for semiconducting SWCNTs ( $\left.E_{\text {act }} \approx 600 \mathrm{meV}[22]\right)$. This may indicate the influence of injection barriers to the charge transport in such systems. It should be noted that for experiments on charge carrier transport at low temperatures, we used gold electrodes in our single-charge carrier devices. However, for device applications, other electrode materials such as ITO, PEDOT:PSS, AI and Ca will be selected in our next research.

\section{Conclusions}

We investigated the effect of SWCNT doping of semiconducting polymer PIDT-BT films. It was shown that adding of $30 \mathrm{wt} \%$ of SWCNT to the PIDT-BT polymer matrix resulted in a decrease in absorbance by a factor of 1.5 without changing of the absorbance edge position. I-Vs of PIDT-BT/SWCNT (1:0.3) nanocomposite film measured at temperatures from 300 to $77 \mathrm{~K}$ in the dark and under illumination by a solar simulator, demonstrated an increase in photocurrent under illumination at $300 \mathrm{~K}$ by factor of ca. 1.4-1.7 with respect to dark $I-V$ s. It was found that the temperature dependence of the resistivity of a lightly doped PIDT-BT/SWCNT nanocomposite film (1:0.1) is determined by the transport of charge carriers in which the thermal activation mechanism prevails; however, for PIDT-BT/SWCNT with intermediate doping (1:0.3), the $\rho(T)$ dependence of the films follows a power law and the mechanism of injection of holes from gold electrodes into our single-carrier device prevails. It has been established that the main mechanisms of charge carrier transfer in such films are SCLC then measured in the dark and photocurrents limited by the space charge under illumination in the temperature range $300-77 \mathrm{~K}$. The obtained nanocomposite films are expected to be useful for various nanotechnology-based hole-only devices.

Acknowledgements The authors are grateful to Professor lain McCulloch for providing the PIDT-BT polymer.

\section{Compliance with ethical standards}

Conflict of interest The authors declare that they have no conflict of interest.

\section{References}

1. Ha C-S (2018) Polymer based hybrid nanocomposites: a progress toward enhancing interfacial Interaction and tailoring advanced applications. Chem Rec 18(7-8):759-775. https://doi. org/10.1002/tcr.201700030
2. Khan W, Sharma R, Saini P (2016) Carbon nanotube-based polymer composites: synthesis, properties and applications. In: Berber MR, Hafez IH (eds) Carbon nanotubes-current progress of their polymer composites. IntechOpen. https://doi. org/10.5772/62497

3. Janas D (2018) Towards monochiral carbon nanotubes: a review of progress in the sorting of single-walled carbon nanotubes. Mater Chem Front 2(1):36-63. https://doi. org/10.1039/C7QM00427C

4. Keru G, Ndungu PG, Nyamori VO (2014) A review on carbon nanotube/polymer composites for organic solar cells. J Energy Res, Int. https://doi.org/10.1002/er.3194

5. Alturaif HA, ALOthman ZA, Shapter JG, Wabaidur SM (2014) Use of carbon nanotubes (CNTs) with polymers in solar cells. Molecules 19(11):17329-17344. https://doi.org/10.3390/ molecules191117329

6. Gabor NM, Zhong Z, Bosnick K, Park J, McEuen PL (2009) Extremely efficient multiple electron-hole pair generation in carbon nanotube photodiodes. Science 325:1367-1371. https ://doi.org/10.1126/science.1176112

7. Chaudhary S, Lu H, Muller AM, Bardeen CJ, Ozkan M (2007) Hierarchical placement and associated optoelectronic impact of carbon nanotubes in polymer-fullerene solar cells. Nano Lett 7:1973-1979. https://doi.org/10.1021/nl070717|

8. Zhang W, Smith J, Watkins SE, Gysel R, McGehee M, Salleo A, Kirkpatrick J, Ashraf S, Anthopoulos T, Heeney M, McCulloch I (2010) Indacenodithiophene semiconducting polymers for high-performance, air-stable transistors. J Am Chem Soc 132(33):11437-11439. https://doi.org/10.1021/ja1049324

9. Wang Y, Michinobu T (2016) Benzothiadiazole and its p-extended, heteroannulated derivatives: useful acceptor building blocks for high-performance donor-acceptor polymers in organic electronics. J Mater Chem C 4:6200-6214. https://doi.org/10.1039/C6TC01860B

10. Zhang Y, Chien SC, Chen KS, Yip HL, Sun Y, Davies JA, Chenb FC, Jen AKY (2011) Increased open circuit voltage in fluorinated benzothiadiazole-based alternating conjugated polymers. Chem Commun 47(39):11026-11028. https://doi.org/10.1039/ C1CC14586J

11. Biswas B, Chowdhury A, Mallik B (2013) Tuning of electrical conductivity and hysteresis effect in poly(methyl methacrylate)-carbon nanotube composite films. RSC Adv. https ://doi.org/10.1039/C2RA22634K

12. Mott NF, Gurney RW (1940) Electronic processes in ionic crystals. Clarendon Press, Oxford

13. Lampert MA, Mark P (1970) Current injection in solids. Academic Press, Cambridge, p 351

14. Mihailetchi VD, Wildeman J, Blom PWM (2005) Space-charge limited photocurrent. Phys Rev Lett 94(12):126602. https:// doi.org/10.1103/PhysRevLett.94.126602

15. Grinberg AA, Luryi S (1987) Space-charge-limited current and capacitance in double-junction diodes. J Appl Phys 61:1181. https://doi.org/10.1063/1.338165

16. Röhr JA, Kirchartz T, Nelson J (2017) On the correct interpretation of the low voltage regime in intrinsic single-carrier devices. J Phys Condens Matter 29(20):205901. https://doi. org/10.1088/1361-648X/aa66cc

17. Röhr JA, Moia D, Haque SA, Kirchartz T, Nelson J (2018) Exploring the validity and limitations of the Mott-Gurney law for charge-carrier mobility determination of semiconducting thin-films. J Phys Cond Matter 30(10):105901. https://doi. org/10.1088/1361-648X/aaabad

18. Mott NF (1969) Conduction in non-crystalline materials. Philos Mag 19:835-852. https://doi.org/10.1080/147864369082163 38 
19. Efros AL, Shklovskii BI (1975) Coulomb gap and low temperature conductivity of disordered systems. J Phys C Solid State Phys 8(11):L49-L51

20. Sheng $P(1980)$ Fluctuation-induced tunneling conduction in disordered materials. Phys Rev B 21(6):2180-2195. https://doi. org/10.1103/PhysRevB.21.2180

21. Aleshin AN (2006) Polymer nanofibers and nanotubes: charge transport and device applications. Adv Mater 18(1):17-27. https ://doi.org/10.1002/adma.200500928

22. Landi BJ, Castro SL, Ruf HJ, Evans CM, Bailey SG, Raffaelle RP (2005) CdSe quantum dot-single wall carbon nanotube complexes for polymeric solar cells. Sol Energy Mater Sol Cells 87(1-4):733-746. https://doi.org/10.1016/j.solmat.2004.07.047

Publisher's Note Springer Nature remains neutral with regard to jurisdictional claims in published maps and institutional affiliations. 